\title{
Some Differential Geometric Relations in the Elastic Shell
}

\author{
Xiaoqin Shen, ${ }^{1}$ Haoming Li, ${ }^{1}$ Kaitai Li, ${ }^{2}$ Xiaoshan Cao, ${ }^{1,3}$ and Qian Yang ${ }^{1}$ \\ ${ }^{1}$ School of Sciences, Xian University of Technology, Xian 710054, China \\ ${ }^{2}$ School of Mathematics and Statistics, Xian Jiaotong University, Xian 710049, China \\ ${ }^{3}$ State Key Laboratory of Transducer Technology, Chinese Academy of Sciences, Shanghai 200050, China \\ Correspondence should be addressed to Xiaoqin Shen; xqshen@xaut.edu.cn
}

Received 30 September 2016; Revised 7 November 2016; Accepted 9 November 2016

Academic Editor: Jose Merodio

Copyright (C) 2016 Xiaoqin Shen et al. This is an open access article distributed under the Creative Commons Attribution License, which permits unrestricted use, distribution, and reproduction in any medium, provided the original work is properly cited.

\begin{abstract}
The theory of the elastic shells is one of the most important parts of the theory of solid mechanics. The elastic shell can be described with its middle surface; that is, the three-dimensional elastic shell with equal thickness comprises a series of overlying surfaces like middle surface. In this paper, the differential geometric relations between elastic shell and its middle surface are provided under the curvilinear coordinate systems, which are very important for forming two-dimensional linear and nonlinear elastic shell models. Concretely, the metric tensors, the determinant of metric matrix field, the Christoffel symbols, and Riemann tensors on the three-dimensional elasticity are expressed by those on the two-dimensional middle surface, which are featured by the asymptotic expressions with respect to the variable in the direction of thickness of the shell. Thus, the novelty of this work is that we can further split three-dimensional mechanics equations into two-dimensional variation problems. Finally, two kinds of special shells, hemispherical shell and semicylindrical shell, are provided as the examples.
\end{abstract}

\section{Introduction}

In $[1,2]$, differential geometric formulae of three-dimensional (3D) domains and two-dimensional (2D) surface are defined in curvilinear ordinates, respectively. Besides, there are some scientists, such as Pobedrya [3], Vekua [4], and Nikabadze [5], who have some contributions in this field. In this paper, we assume that the three-dimensional elastic shell with equal thickness comprises a series of overlying surfaces like middle surface. Thus, the differential geometric relations between 3D elasticity and 2D middle surface are provided which are very important for forming 2D shell model from 3D equations (cf. [6-9]). Concretely, the metric tensor, the determinant of metric matrix field, the Christoffel symbols, and Riemann tensors on the 3D domain are expressed by those on the 2D surface, which are featured by the asymptotic expressions with respect to the variable in the direction of thickness of the shell. In Section 3, two kinds of special shells, that is, hemispherical shell and semicylindrical shell, are provided as the examples.

In this section, we mainly introduce some notations. Our notations are essentially borrowed from [2]. In what follows, Latin indices and exponents $i, j, k, \ldots$ take their values in the set $\{1,2,3\}$, whereas Greek indices and exponents $\alpha, \beta, \gamma, \ldots$ take their values in the set $\{1,2\}$. In addition, the repeated index summation convention is systematically used. The Euclidean scalar product and the exterior product of $\vec{a}, \vec{b} \in \mathbb{R}^{3}$ are noted by $\vec{a} \cdot \vec{b}$ and $\vec{a} \times \vec{b}$, respectively.

Let $\omega$ (cf. Figure 1) be an open, bounded, connected subset of $\mathbb{R}^{2}$, the boundary $\gamma=\partial \omega$ of which is Lipschitz-continuous, and let $\gamma=\gamma_{0} \cup \gamma_{1}$ with $\gamma_{0} \cap \gamma_{1}=\emptyset$. Let $y=\left(y_{\alpha}\right)$ denote a generic point in the set $\bar{\omega}$ (i.e., closure of $\omega$ ) and let $\partial_{\alpha}:=$ $\partial / \partial y_{\alpha}$. Let there be given an injective mapping $\vec{\theta} \in C^{3}\left(\bar{\omega} ; \mathbb{R}^{3}\right)$, such that the two vectors

$$
\vec{a}_{\alpha}(y):=\partial_{\alpha} \vec{\theta}(y)
$$

are linearly independent at all points $y \in \bar{\omega}$. These two vectors thus span the tangent plane to the surface

$$
S:=\vec{\theta}(\bar{\omega})
$$

at the point $\vec{\theta}(y)$, and the unit vector

$$
\vec{a}_{3}(y):=\frac{\vec{a}_{1}(y) \times \vec{a}_{2}(y)}{\left|\vec{a}_{1}(y) \times \vec{a}_{2}(y)\right|}
$$




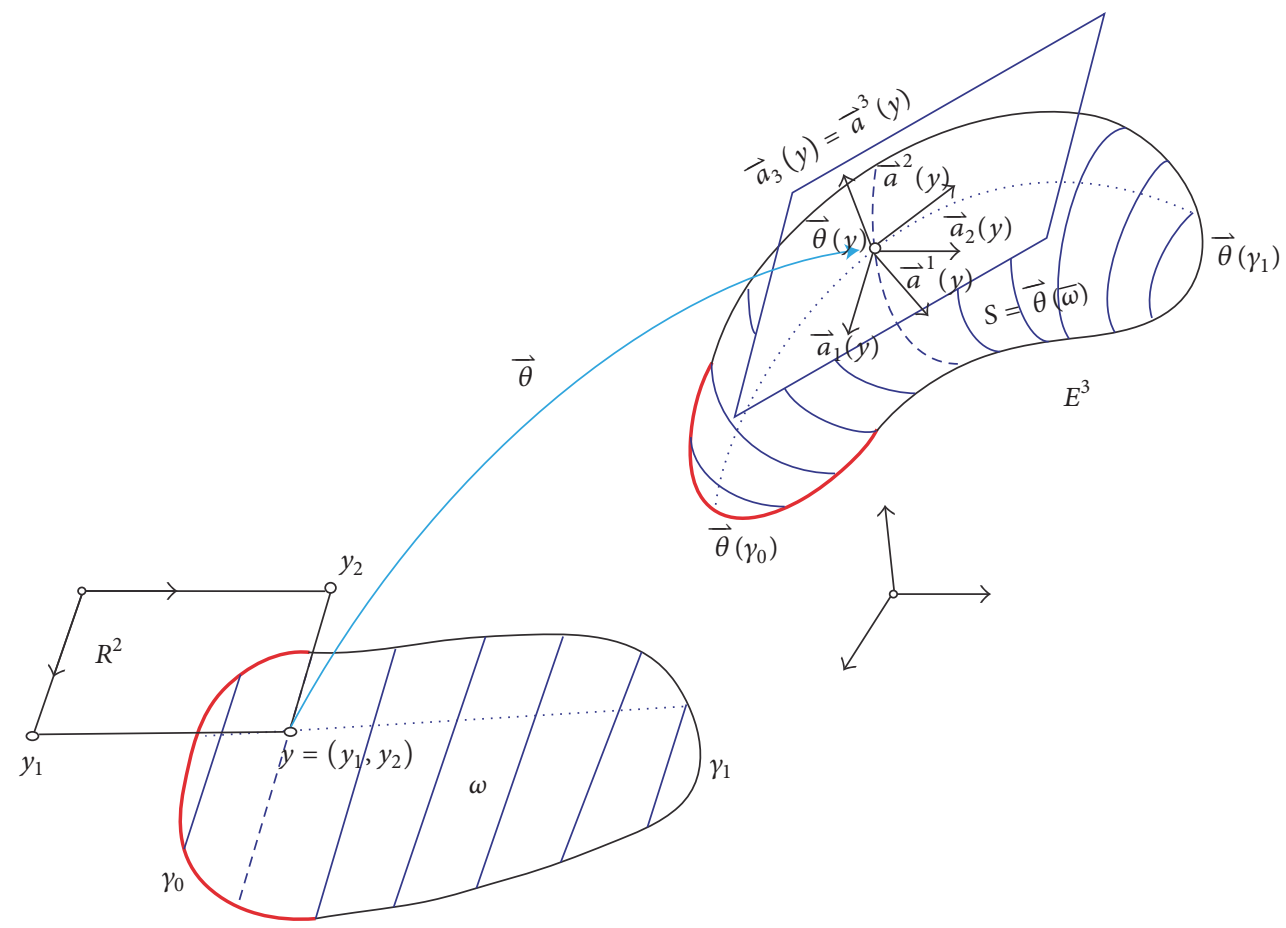

FIgURE 1: Two-dimensional domain $\omega$ and surface $S$ (cf. [2]).

is normal to $S$ at the point $\vec{\theta}(y)$. These vectors $\vec{a}_{i}(y)$ constitute the covariant basis at the point $\theta(y)$, whereas the vectors $\vec{a}^{i}(y)$ defined by the relations

$$
\vec{a}^{i}(y) \cdot \vec{a}_{j}(y)=\delta_{j}^{i}
$$

constitute the contravariant basis at the point $\theta(y)$, where $\delta_{j}^{i}$ is the Kronecker symbol (note that $\vec{a}^{3}(y)=\vec{a}_{3}(y)$ and the vector $\vec{a}^{\alpha}(y)$ is also in the tangent plane to $S$ at $\left.\vec{\theta}(y)\right)$ (cf. Figure 1).

The covariant and contravariant components $a_{\alpha \beta}$ and $a^{\alpha \beta}$ of the metric tensor of $S$, the Christoffel symbol $\Gamma_{\alpha \beta, \sigma}^{*}$ on $S$, the covariant and mixed components $b_{\alpha \beta}$ and $b_{\alpha}^{\beta}$ of the curvature tensor of $S$, and the covariant of the third fundament form on $S$ are then defined as follows (the explicit dependence on the variable $y \in \bar{\omega}$ is henceforth dropped):

$$
\begin{aligned}
a_{\alpha \beta} & :=\vec{a}_{\alpha} \cdot \vec{a}_{\beta}, \\
a^{\alpha \beta} & :=\vec{a}^{\alpha} \cdot \vec{a}^{\beta}, \\
\left(a^{\alpha \beta}\right) & =\left(a_{\alpha \beta}\right)^{-1}, \\
\vec{a}^{\alpha} & =a^{\alpha \beta} \vec{a}_{\beta}, \\
\Gamma_{\alpha \beta, \sigma}^{*} & :=\vec{a}_{\sigma} \cdot \partial_{\alpha} \vec{a}_{\beta}, \\
\Gamma_{\alpha \beta}^{* \sigma} & :=\vec{a}^{\sigma} \cdot \partial_{\alpha} \vec{a}_{\beta}, \\
b_{\alpha \beta} & :=\vec{a}_{3} \cdot \partial_{\alpha} \vec{a}_{\beta}, \\
b_{\alpha}^{\beta} & :=a^{\beta \sigma} b_{\sigma \alpha}, \\
c_{\alpha \beta} & :=\partial_{\alpha} \vec{a}_{3} \cdot \partial_{\beta} \vec{a}_{3},
\end{aligned}
$$

where $\left(a_{\alpha \beta}\right)$ is symmetric and positive-definite matrix field, $\left(b_{\alpha \beta}\right)$ and $\left(c_{\alpha \beta}\right)$ are symmetric matrix fields. The determinants of metric tensor, curvature tensor, and the third fundament form are

$$
\begin{aligned}
& a:=\operatorname{det}\left(a_{\alpha \beta}\right), \\
& b:=\operatorname{det}\left(b_{\alpha \beta}\right), \\
& c:=\operatorname{det}\left(c_{\alpha \beta}\right) .
\end{aligned}
$$

Thus, the Riemann tensors on the middle surface $S$ are defined by (cf. [10])

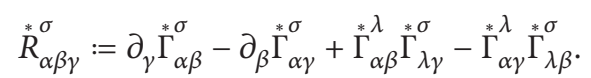

Then, the covariant components of Riemann tensors on $S$ are defined by

$$
\stackrel{*}{R}_{\alpha \beta \lambda \sigma}:=a_{\gamma \beta} R_{\alpha \lambda \sigma}^{\gamma} .
$$

Assume that there is a shell $\widehat{\Omega}^{\varepsilon}$ (cf. Figure 2) with middle surface $S=\vec{\theta}(\bar{\omega})$ and whose thickness $2 \varepsilon>0$ is arbitrarily small. Hence, for each $\varepsilon>0$, the reference configuration of the shell is $\widehat{\Omega}^{\varepsilon}=\vec{\Theta}\left(\bar{\Omega}^{\varepsilon}\right)$, where $\bar{\Omega}^{\varepsilon}=\bar{\omega} \times[-\varepsilon, \varepsilon]$; that is,

$$
\vec{\Theta}(y, \xi)=\vec{\theta}(y)+\xi \vec{a}_{3}(y), \quad-\varepsilon \leq \xi \leq \varepsilon .
$$

In this sense, the 3D elastic shell with equal thickness comprises a series of overlying surfaces like middle surface. The top and bottom faces of $\vec{\Theta}\left(\bar{\Omega}^{\varepsilon}\right)$ are $\Gamma_{t}=\vec{\Theta}(\omega \times\{+\varepsilon\})$ and $\Gamma_{b}=\vec{\Theta}(\omega \times\{-\varepsilon\})$. The lateral face is $\Gamma_{l}=\Gamma_{0} \cup \Gamma_{1}$, where 


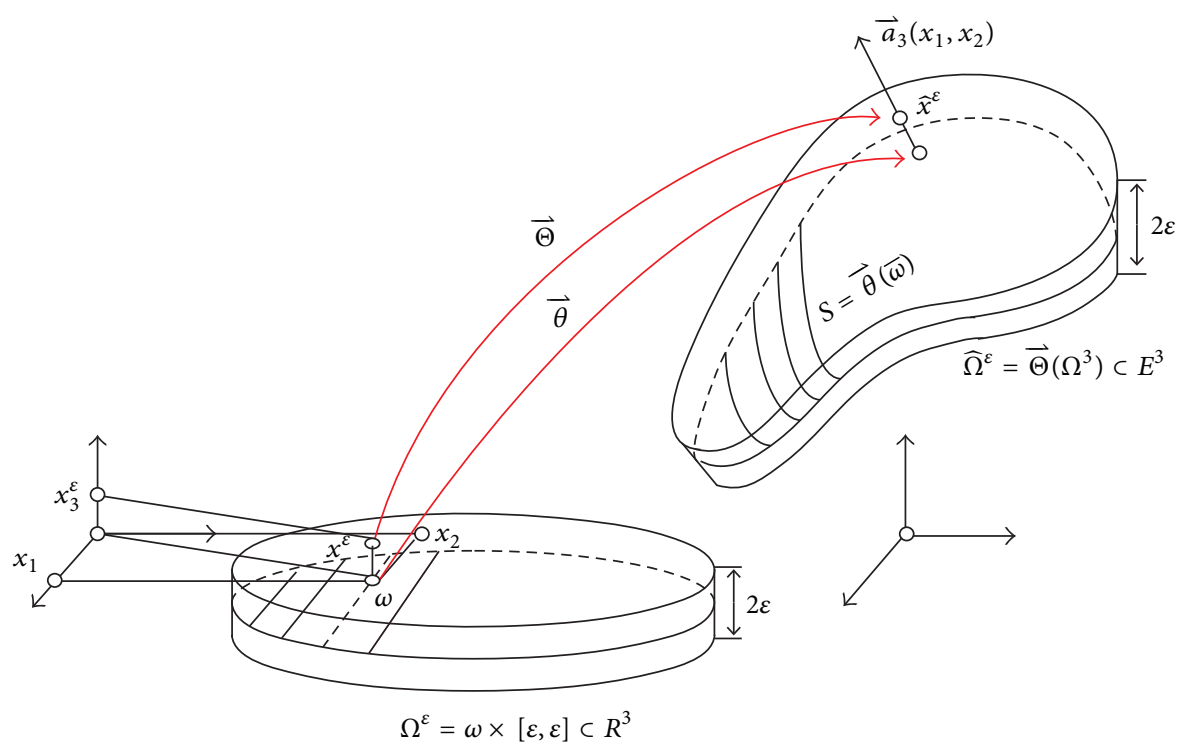

FIgURE 2: The shell $\widehat{\Omega}^{\varepsilon}$ with middle surface $S$ (cf. [2]).

$\Gamma_{0}=\vec{\theta}\left(\gamma_{0}\right) \times[-\varepsilon,+\varepsilon], \Gamma_{1}=\vec{\theta}\left(\gamma_{1}\right) \times[-\varepsilon,+\varepsilon]$ (cf. [11]). Let $x=\left(x_{i}\right)=\left(y_{1}, y_{2}, \xi\right)$ denote a generic point in the set $\bar{\Omega}^{\varepsilon}$. The mapping $\vec{\Theta}: \bar{\Omega}^{\varepsilon} \rightarrow R^{3}$ is injective and the three vectors

$$
\vec{g}_{i}(x):=\partial_{i} \vec{\Theta}(x)
$$

are linearly independent at all points $x \in \bar{\Omega}^{\varepsilon}$. The vectors $\vec{g}^{i}(y)$ are defined by the relations

$$
\vec{g}^{i}(x) \cdot \vec{g}_{j}(x)=\delta_{j}^{i} .
$$

These relations constitute the contravariant basis at the point $\vec{\Theta}(x) \in S$. The covariant and contravariant components $g_{i j}$ and $g^{i j}$ of the metric tensor of $\vec{\Theta}\left(\bar{\Omega}^{\varepsilon}\right)$, the Christoffel symbols $\Gamma_{i j, k}$ and $\Gamma_{i j}^{k}$ on $\vec{\Theta}\left(\bar{\Omega}^{\varepsilon}\right)$ are then defined as follows (the explicit dependence on the variable $x \in \bar{\Omega}$ is henceforth dropped):

$$
\begin{aligned}
g_{i j} & :=\vec{g}_{i} \cdot \vec{g}_{j}, \\
g^{i j} & :=\vec{g}^{i} \cdot \vec{g}^{j}, \\
\Gamma_{i j, k} & :=\vec{g}_{k} \cdot \partial_{i} \vec{g}_{j}, \\
\Gamma_{i j}^{k} & =g^{k l} \Gamma_{i j, l} .
\end{aligned}
$$

The determinant of metric tensor is

$$
g:=\operatorname{det}\left(g_{i j}\right) \text {. }
$$

Thus, the Riemann tensors on $\vec{\Theta}\left(\bar{\Omega}^{\varepsilon}\right)$ are defined by

$$
R_{i k j}^{p}:=\partial_{\gamma} \Gamma_{i j}^{p}-\partial_{\beta} \Gamma_{i k}^{p}+\Gamma_{i j}^{q} \Gamma_{q k}^{p}-\Gamma_{i k}^{q} \Gamma_{q j}^{p} .
$$

Then, the covariant components of Riemann tensors on $\vec{\Theta}\left(\bar{\Omega}^{\varepsilon}\right)$ are defined by

$$
R_{i l j k}:=g_{l p} R_{i j k}^{p} .
$$

\section{Main Results}

Theorem 1. Assume that there is a shell with middle surface $S=\vec{\theta}(\bar{\omega})$ whose thickness $2 \varepsilon>0$ is arbitrarily small, where $\omega$ is open, bounded, and connected in $\mathbb{R}^{2}$ with Lipschitz-continuous boundary $\gamma=\partial \omega$ and $\vec{\theta} \in C^{3}\left(\bar{\omega} ; \mathbb{R}^{3}\right)$. Hence, for each $\varepsilon>0$, the reference configuration of the shell is $\vec{\Theta}(\bar{\Omega})$, where $\bar{\Omega}=\bar{\omega} \times$ $[-\varepsilon, \varepsilon]$; that is,

$$
\vec{\Theta}(y, \xi)=\vec{\theta}(y)+\xi \vec{a}_{3}(y) .
$$

The metric tensors on $\vec{\Theta}(y, \xi)$ and $\vec{\theta}\left(\bar{\omega}^{\varepsilon}\right)$ are $g_{i j}$ and $a_{\alpha \beta}$, respectively. $b_{\alpha \beta}$ and $c_{\alpha \beta}$ are the second and third fundamental forms on $\vec{\theta}(\bar{\omega})$. Then, the following differential geometric relations hold:

$$
\begin{aligned}
& g_{\alpha \beta}=a_{\alpha \beta}-2 \xi b_{\alpha \beta}+\xi^{2} c_{\alpha \beta}, \\
& g_{\alpha 3}=g_{3 \alpha}=0, \\
& g_{33}=1,
\end{aligned}
$$

$$
\alpha, \beta=1,2, \xi \in[-\varepsilon, \varepsilon]
$$

Proof.

$$
\begin{aligned}
g_{\alpha \beta}= & \vec{g}_{\alpha} \cdot \vec{g}_{\beta}=\partial_{\alpha} \vec{\Theta} \cdot \partial_{\beta} \vec{\Theta} \\
= & \partial_{\alpha}\left(\vec{\theta}+\xi \vec{a}_{3}\right) \cdot \partial_{\beta}\left(\vec{\theta}+\xi \vec{a}_{3}\right) \\
= & \partial_{\alpha} \vec{\theta} \cdot \partial_{\beta} \vec{\theta}+\partial_{\alpha} \vec{\theta} \cdot \partial_{\beta}\left(\xi \vec{a}_{3}\right)+\partial_{\alpha}\left(\xi \vec{a}_{3}\right) \cdot \partial_{\beta} \vec{\theta} \\
& +\partial_{\alpha}\left(\xi \vec{a}_{3}\right) \cdot \partial_{\beta}\left(\xi \vec{a}_{3}\right) \\
= & \vec{a}_{\alpha} \cdot \vec{a}_{\beta}+\xi \vec{a}_{\alpha} \cdot \partial_{\beta} \vec{a}_{3}+\xi \partial_{\alpha} \vec{a}_{3} \cdot \vec{a}_{\beta}+\xi^{2} \partial_{\alpha} \vec{a}_{3} \\
& \cdot \partial_{\beta} \vec{a}_{3} .
\end{aligned}
$$


Submitting (1) and (5)-(7) into (20), based on the symmetry of $b_{\alpha \beta}$, we have

$$
\begin{aligned}
g_{\alpha \beta} & =a_{\alpha \beta}-2 \xi b_{\alpha \beta}+\xi^{2} c_{\alpha \beta}, \\
g_{3 \alpha} & =\vec{g}_{3} \cdot \vec{g}_{\alpha}=\partial_{3} \vec{\Theta} \cdot \partial_{\alpha} \vec{\Theta} \\
& =\partial_{3}\left(\vec{\theta}+\xi \vec{a}_{3}\right) \cdot \partial_{\alpha}\left(\vec{\theta}+\xi \vec{a}_{3}\right)=\vec{a}_{3} \cdot \partial_{\alpha}\left(\vec{\theta}+\xi \vec{a}_{3}\right) \\
& =\vec{a}_{3} \cdot \partial_{\alpha} \vec{\theta}+\vec{a}_{3} \cdot \partial_{\alpha}\left(\xi \vec{a}_{3}\right)=\vec{a}_{3} \cdot \vec{a}_{\alpha}+\xi \vec{a}_{3} \cdot \partial_{\alpha} \vec{a}_{3} .
\end{aligned}
$$

From the definition of $a_{3}$, we know

$$
\begin{aligned}
& \vec{a}_{3} \cdot \vec{a}_{\alpha}=0, \\
& \vec{a}_{3} \cdot \vec{a}_{3}=1 .
\end{aligned}
$$

Then,

$$
\partial_{\alpha}\left(\vec{a}_{3} \cdot \vec{a}_{3}\right)=2 \vec{a}_{3} \cdot \partial_{\alpha} \vec{a}_{3}=0
$$

Thus,

$$
\vec{a}_{3} \cdot \partial_{\alpha} \vec{a}_{3}=0
$$

Submitting (23)-(25) into (22), we get

$$
g_{3 \alpha}=0 \text {. }
$$

Similarly,

$$
\begin{aligned}
g_{\alpha 3}= & 0, \\
g_{33}= & \vec{g}_{3} \cdot \vec{g}_{3}=\partial_{3} \vec{\Theta} \cdot \partial_{3} \vec{\Theta} \\
= & \partial_{3}\left(\vec{\theta}+\xi \vec{a}_{3}\right) \cdot \partial_{3}\left(\vec{\theta}+\xi \vec{a}_{3}\right) \\
= & \partial_{3} \vec{\theta} \cdot \partial_{3} \vec{\theta}+\partial_{3} \vec{\theta} \cdot \partial_{3}\left(\xi \vec{a}_{3}\right)+\partial_{3}\left(\xi \vec{a}_{3}\right) \cdot \partial_{3} \vec{\theta} \\
& +\partial_{3}\left(\xi \vec{a}_{3}\right) \cdot \partial_{3}\left(\xi \vec{a}_{3}\right)=0+0+0+\vec{a}_{3} \cdot \vec{a}_{3}=1 .
\end{aligned}
$$

Since $\left(g^{i j}\right)=\left(g_{i j}\right)^{-1}$, the contravariant components of $g^{i j}$ should be expressed as follows.

Theorem 2. Under the assumptions of Theorem 1, let $g^{i j}$ be the contravariant components of the metric tensors on $\vec{\Theta}(y, \xi)$. Then, the following formulae hold:

$$
\begin{aligned}
& g^{11}=g^{-1}\left(a_{22}-2 \xi b_{22}+\xi^{2} c_{22}\right), \\
& g^{12}=g^{21}=-g^{-1}\left(a_{12}-2 \xi b_{12}+\xi^{2} c_{12}\right), \\
& g^{22}=g^{-1}\left(a_{11}-2 \xi b_{11}+\xi^{2} c_{11}\right), \\
& g^{\alpha 3}=g^{3 \alpha}=0, \\
& g^{33}=1,
\end{aligned}
$$

where $g=\operatorname{det}\left(g_{i j}\right)=\left(a_{11}-2 \xi b_{11}+\xi^{2} c_{11}\right)\left(a_{22}-2 \xi b_{22}+\xi^{2} c_{22}\right)-$ $\left(a_{12}-2 \xi b_{12}+\xi^{2} c_{12}\right)^{2}$.
Proof.

$$
\left(g_{i j}\right)^{-1}=\left(\begin{array}{cc}
\left(g_{\alpha \beta}\right) & 0 \\
0 & g_{33}
\end{array}\right)^{-1}=\left(\begin{array}{cc}
\left(g_{\alpha \beta}\right)^{-1} & 0 \\
0 & \left(g_{33}\right)^{-1}
\end{array}\right),
$$

where

$$
\left(g_{\alpha \beta}\right)^{-1}=\left(\begin{array}{ll}
g_{11} & g_{12} \\
g_{21} & g_{22}
\end{array}\right)^{-1}=g^{-1}\left(\begin{array}{cc}
g_{22} & -g_{12} \\
-g_{21} & g_{11}
\end{array}\right) .
$$

Since $\left(g^{i j}\right)=\left(g_{i j}\right)^{-1}$, formula (28) can be derived easily.

Theorem 3. Under the assumptions of Theorem 1, let $\Gamma_{i j, k}$ and $\stackrel{*}{\Gamma}_{\alpha \beta, \gamma}$ be the Christoffel symbols on $\vec{\Theta}(y, \xi)$ and $\vec{\theta}(\bar{\omega})$, respectively. Then, the following formulae hold:

$$
\begin{aligned}
\Gamma_{\alpha \beta, \sigma}= & \Gamma_{\alpha \beta, \sigma}^{*}+\xi \vec{a}_{\sigma} \cdot \partial_{\alpha \beta} \vec{a}_{3}+\xi \partial_{\sigma} \vec{a}_{3} \cdot \partial_{\alpha} \vec{a}_{\beta}+\xi^{2} \partial_{\sigma} \vec{a}_{3} \\
& \cdot \partial_{\alpha \beta} \vec{a}_{3}, \\
\Gamma_{\alpha \beta, 3}= & b_{\alpha \beta}-\xi c_{\alpha \beta}, \\
\Gamma_{\alpha 3, \sigma}= & \Gamma_{3 \alpha, \sigma}=-b_{\alpha \sigma}+\xi c_{\alpha \sigma}, \\
\Gamma_{33, \alpha}= & \Gamma_{\alpha 3,3}=\Gamma_{3 \alpha, 3}=\Gamma_{33,3}=0,
\end{aligned}
$$

$\alpha, \beta, \sigma=1,2$.

Proof.

$$
\begin{aligned}
\Gamma_{\alpha \beta, \sigma}= & \vec{g}_{\sigma} \cdot \partial_{\alpha} \vec{g}_{\beta}=\partial_{\sigma} \vec{\Theta} \cdot \partial_{\alpha}\left(\partial_{\beta} \vec{\Theta}\right) \\
= & \partial_{\sigma}\left(\vec{\theta}+\xi \vec{a}_{3}\right) \cdot \partial_{\alpha \beta}\left(\vec{\theta}+\xi \vec{a}_{3}\right) \\
= & \partial_{\sigma} \vec{\theta} \cdot \partial_{\alpha \beta} \vec{\theta}+\partial_{\sigma} \vec{\theta} \cdot \partial_{\alpha \beta}\left(\xi \vec{a}_{3}\right)+\partial_{\sigma}\left(\xi \vec{a}_{3}\right) \cdot \partial_{\alpha \beta} \vec{\theta} \\
& +\partial_{\sigma}\left(\xi \vec{a}_{3}\right) \cdot \partial_{\alpha \beta}\left(\xi \vec{a}_{3}\right) \\
= & \Gamma_{\alpha \beta, \sigma}^{*}+\xi \vec{a}_{\sigma} \cdot \partial_{\alpha \beta} \vec{a}_{3}+\xi \partial_{\sigma} \vec{a}_{3} \cdot \partial_{\alpha} \vec{a}_{\beta}+\xi^{2} \partial_{\sigma} \vec{a}_{3} \\
& \cdot \partial_{\alpha \beta} \vec{a}_{3}, \\
\Gamma_{\alpha \beta, 3}= & \vec{g}_{3} \cdot \partial_{\alpha} \vec{g}_{\beta}=\partial_{3} \vec{\Theta} \cdot \partial_{\alpha}\left(\partial_{\beta} \vec{\Theta}\right) \\
= & \partial_{3}\left(\vec{\theta}+\xi \vec{a}_{3}\right) \cdot \partial_{\alpha \beta}\left(\vec{\theta}+\xi \vec{a}_{3}\right) \\
= & \vec{a}_{3} \cdot \partial_{\alpha \beta}\left(\vec{\theta}+\xi \vec{a}_{3}\right)=\vec{a}_{3} \cdot \partial_{\alpha \beta} \vec{\theta}+\xi \vec{a}_{3} \cdot \partial_{\alpha \beta} \vec{a}_{3} .
\end{aligned}
$$

Since $\left(\vec{a}_{3}, \vec{a}_{3}\right)=1$, we have

$$
\begin{aligned}
\partial_{\alpha \beta}\left(\vec{a}_{3}, \vec{a}_{3}\right) & =\partial_{\alpha}\left(\partial_{\beta}\left(\vec{a}_{3}, \vec{a}_{3}\right)\right)=\partial_{\alpha}\left(2 \vec{a}_{3} \cdot \partial_{\beta} \vec{a}_{3}\right) \\
& =2 \partial_{\alpha} \vec{a}_{3} \cdot \partial_{\beta} \vec{a}_{3}+2 \vec{a}_{3} \cdot \partial_{\alpha \beta} \vec{a}_{3}=0 .
\end{aligned}
$$

Thus,

$$
\vec{a}_{3} \cdot \partial_{\alpha \beta} \vec{a}_{3}=-\partial_{\alpha} \vec{a}_{3} \cdot \partial_{\beta} \vec{a}_{3}
$$


Submitting (35) and (7) into (33), we get

$$
\begin{aligned}
\Gamma_{\alpha \beta, 3} & =b_{\alpha \beta}-\xi c_{\alpha \beta}, \\
\Gamma_{\alpha 3, \sigma} & =\vec{g}_{\sigma} \cdot \partial_{\alpha} \vec{g}_{3}=\partial_{\sigma} \vec{\Theta} \cdot \partial_{\alpha}\left(\partial_{3} \vec{\Theta}\right) \\
& =\partial_{\sigma}\left(\vec{\theta}+\xi \vec{a}_{3}\right) \cdot \partial_{\alpha}\left(\partial_{3}\left(\vec{\theta}+\xi \vec{a}_{3}\right)\right) \\
& =\partial_{\sigma}\left(\vec{\theta}+\xi \vec{a}_{3}\right) \cdot \partial_{\alpha} \vec{a}_{3} \\
& =\partial_{\sigma} \vec{\theta} \cdot \partial_{\alpha} \vec{a}_{3}+\xi \partial_{\sigma} \vec{a}_{3} \cdot \partial_{\alpha} \vec{a}_{3}=-b_{\alpha \sigma}+\xi c_{\alpha \sigma} .
\end{aligned}
$$

Similarly,

$$
\begin{aligned}
\Gamma_{3 \alpha, \sigma} & =-b_{\alpha \sigma}+\xi c_{\alpha \sigma}, \\
\Gamma_{33, \alpha} & =\vec{g}_{\alpha} \cdot \partial_{3} \vec{g}_{3}=\partial_{\alpha} \vec{\Theta} \cdot \partial_{3}\left(\partial_{3} \vec{\Theta}\right) \\
& =\partial_{\alpha}\left(\vec{\theta}+\xi \vec{a}_{3}\right) \cdot \partial_{3}\left(\partial_{3}\left(\vec{\theta}+\xi \vec{a}_{3}\right)\right) \\
& =\partial_{\alpha}\left(\vec{\theta}+\xi \vec{a}_{3}\right) \cdot \partial_{3} \vec{a}_{3}=0, \\
\Gamma_{\alpha 3,3} & =\vec{g}_{3} \cdot \partial_{\alpha} \vec{g}_{3}=\partial_{3} \vec{\Theta} \cdot \partial_{\alpha}\left(\partial_{3} \vec{\Theta}\right) \\
& =\partial_{3}\left(\vec{\theta}+\xi \vec{a}_{3}\right) \cdot \partial_{\alpha}\left(\partial_{3}\left(\vec{\theta}+\xi \vec{a}_{3}\right)\right)=\vec{a}_{3} \cdot \partial_{\alpha} \vec{a}_{3} \\
& =0, \\
\Gamma_{33,3} & =\vec{g}_{3} \cdot \partial_{3} \vec{g}_{3}=\partial_{3} \vec{\Theta} \cdot \partial_{3}\left(\partial_{3} \vec{\Theta}\right) \\
& =\partial_{3}\left(\vec{\theta}+\xi \vec{a}_{3}\right) \cdot \partial_{3}\left(\partial_{3}\left(\vec{\theta}+\xi \vec{a}_{3}\right)\right)=\vec{a}_{3} \cdot \partial_{3} \vec{a}_{3} \\
& =0 .
\end{aligned}
$$

Thus, the Christoffel symbols $\Gamma_{i j}^{k}$ and $\stackrel{*}{\Gamma}_{\alpha \beta}^{\sigma}$ have similar relations.

Theorem 4. Under the assumptions of Theorem 1 , let $\Gamma_{i j}^{k}$ be the Christoffel symbols on $\vec{\Theta}(y, \xi)$. Then, the following formulae hold:

$$
\begin{array}{ll}
\Gamma_{\alpha \beta}^{\sigma} & =g^{\sigma \tau}\left(\stackrel{*}{\Gamma}_{\alpha \beta, \tau}+\xi \vec{a}_{\tau} \cdot \partial_{\alpha \beta} \vec{a}_{3}+\xi \partial_{\tau} \vec{a}_{3} \cdot \partial_{\alpha} \vec{a}_{\beta}+\xi^{2} \partial_{\tau} \vec{a}_{3}\right. \\
\cdot & \left.\partial_{\alpha \beta} \vec{a}_{3}\right), \\
\Gamma_{\alpha \beta}^{3}=b_{\alpha \beta}-\xi c_{\alpha \beta}, & \\
\Gamma_{\alpha 3}^{\sigma}=\Gamma_{3 \alpha}^{\sigma}=g^{\sigma \tau}\left(-b_{\alpha \tau}+\xi c_{\alpha \tau}\right), & \alpha, \beta, \sigma=1,2 . \\
\Gamma_{33}^{\alpha}=\Gamma_{\alpha 3}^{3}=\Gamma_{3 \alpha}^{3}=\Gamma_{33}^{3}=0, &
\end{array}
$$

Proof. Because of (13), we have

$$
\begin{aligned}
& \Gamma_{\alpha \beta}^{\sigma}=g^{\sigma l} \Gamma_{\alpha \beta, l}=g^{\sigma \tau} \Gamma_{\alpha \beta, \tau}+g^{\sigma 3} \Gamma_{\alpha \beta, 3}=g^{\sigma \tau} \Gamma_{\alpha \beta, \tau}, \\
& \Gamma_{\alpha \beta}^{3}=g^{3 l} \Gamma_{\alpha \beta, l}=g^{31} \Gamma_{\alpha \beta, 1}+g^{32} \Gamma_{\alpha \beta, 2}+g^{33} \Gamma_{\alpha \beta, 3}=\Gamma_{\alpha \beta, 3}, \\
& \Gamma_{\alpha 3}^{\sigma}=\Gamma_{3 \alpha}^{\sigma}=g^{\sigma l} \Gamma_{\alpha 3, l}=g^{\sigma \tau} \Gamma_{\alpha 3, \tau}+g^{\sigma 3} \Gamma_{\alpha 3,3}=g^{\sigma \tau} \Gamma_{\alpha 3, \tau},
\end{aligned}
$$

$$
\begin{aligned}
& \Gamma_{33}^{\alpha}=g^{\alpha l} \Gamma_{33, l}=g^{\alpha \tau} \Gamma_{33, \tau}+g^{\alpha 3} \Gamma_{33,3}=0, \\
& \Gamma_{\alpha 3}^{3}=\Gamma_{3 \alpha}^{3}=g^{3 l} \Gamma_{\alpha 3, l}=g^{3 \tau} \Gamma_{\alpha 3, \tau}+g^{33} \Gamma_{\alpha 3,3}=\Gamma_{\alpha 3,3}, \\
& \Gamma_{33}^{3}=g^{3 l} \Gamma_{33, l}=g^{3 \tau} \Gamma_{33, \tau}+g^{33} \Gamma_{33,3}=\Gamma_{33,3} .
\end{aligned}
$$

Thus, formula (38) can be derived easily from the results of Theorems 2 and 3.

Theorem 5. Under the assumptions of Theorem 1 , let $R_{i k j}^{p}, R_{i l k j}$ and $\stackrel{*}{R}_{\alpha \gamma \beta}^{\sigma}, \stackrel{*}{R}_{\alpha \lambda \gamma \beta}$ be the Riemann tensors on $\vec{\Theta}(y, \xi)$ and $\vec{\theta}(y)$, respectively. Then, the following formulae hold:

$$
\begin{aligned}
& R_{i k j}^{p}=0, \\
& R_{i l k j}=0, \quad i, j, k, p, l=1,2,3 ; \\
& \stackrel{*}{R_{\alpha \gamma \beta}^{\sigma}}=b_{\alpha \beta} b_{\gamma}^{\sigma}-b_{\alpha \gamma} b_{\beta}^{\sigma}, \\
& \stackrel{*}{R_{\alpha \lambda \gamma \beta}}=b_{\alpha \beta} b_{\lambda \gamma}-b_{\alpha \gamma} b_{\beta \lambda}, \\
& \quad \alpha, \beta, \sigma, \gamma, \lambda=1,2 .
\end{aligned}
$$

Proof. As we all know, formula (40) has been proven by Ciarlet in [12] (cf. Theorem 1.6-1). We only should prove formula (41).

From Gaussian formula of coordinate systems (cf. [7]), we have

$$
\begin{aligned}
\partial_{\beta} \vec{a}_{\alpha} & =\left(\partial_{\beta} \vec{a}_{\alpha} \cdot \vec{a}^{\lambda}\right) \vec{a}_{\lambda}+\left(\partial_{\beta} \vec{a}_{\alpha} \cdot \vec{a}^{3}\right) \vec{a}_{3} \\
& =\stackrel{*}{\Gamma}_{\alpha \beta}^{\lambda} \vec{a}_{\lambda}+b_{\alpha \beta} \vec{a}_{3} .
\end{aligned}
$$

Submitting $\partial_{\lambda} \vec{a}_{3}=-b_{\lambda}^{\sigma} \vec{a}_{\sigma}$ into (42), we have

$$
\begin{aligned}
\partial_{\beta \gamma} \vec{a}_{\alpha}= & \left(\partial_{\gamma} \stackrel{*}{\Gamma}_{\alpha \beta}^{\lambda}\right) \vec{a}_{\lambda}+\stackrel{*}{\Gamma}_{\alpha \beta}^{\lambda} \partial_{\gamma} \vec{a}_{\lambda}+\left(\partial_{\gamma} b_{\alpha \beta}\right) \vec{a}_{3} \\
& +b_{\alpha \beta} \partial_{\gamma} \vec{a}_{3} .
\end{aligned}
$$

Submitting (42) into (43), we have

$$
\begin{aligned}
\partial_{\beta \gamma} \vec{a}_{\alpha}= & \left(\partial_{\gamma} \stackrel{*}{\Gamma}_{\alpha \beta}^{\sigma}+\stackrel{*}{\Gamma}_{\alpha \beta}^{\lambda} \stackrel{*}{\Gamma}_{\lambda \gamma}^{\sigma}-b_{\alpha \beta} b_{\gamma}^{\sigma}\right) \vec{a}_{\sigma} \\
& +\left(\stackrel{*}{\Gamma}_{\alpha \beta}^{\lambda} b_{\gamma \lambda}+\partial_{\gamma} b_{\alpha \beta}\right) \vec{a}_{3} .
\end{aligned}
$$

Similarly,

$$
\begin{aligned}
\partial_{\gamma \beta} \vec{a}_{\alpha}= & \left(\partial_{\beta} \stackrel{*}{\Gamma}_{\alpha \gamma}^{\sigma}+\stackrel{*}{\Gamma}_{\alpha \gamma}^{\lambda} \stackrel{*}{\Gamma}_{\lambda \beta}^{\sigma}-b_{\alpha \gamma} b_{\beta}^{\sigma}\right) \vec{a}_{\sigma} \\
& +\left(\stackrel{*}{\Gamma}_{\alpha \gamma}^{\lambda} b_{\beta \lambda}+\partial_{\beta} b_{\alpha \gamma}\right) \vec{a}_{3} .
\end{aligned}
$$




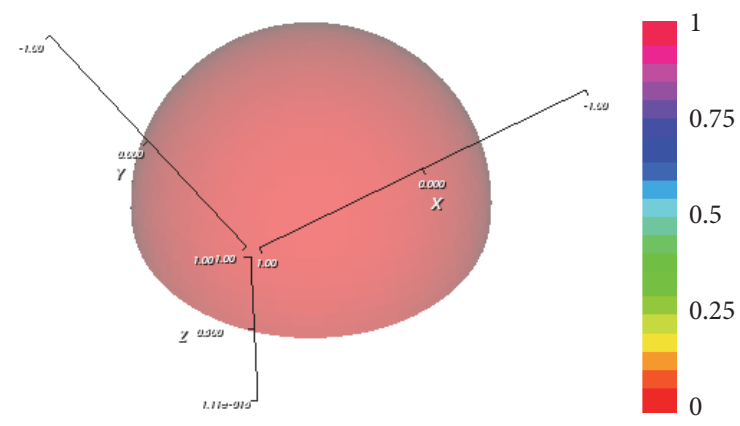

FIGURE 3: Middle surface of hemispherical shell.

Because of $\partial_{\beta \gamma} \vec{a}_{\alpha}=\partial_{\gamma \beta} \vec{a}_{\alpha}$, we can deduce by (44)-(45) that

$$
\begin{aligned}
0= & \left(\partial_{\gamma} \stackrel{*}{\Gamma}_{\alpha \beta}^{*}-\partial_{\beta} \stackrel{*}{\Gamma}_{\alpha \gamma}^{\sigma}+\stackrel{*}{\Gamma}_{\alpha \beta}^{* \lambda} \Gamma_{\lambda \gamma}^{* \sigma}-\stackrel{*}{\Gamma}_{\alpha \gamma}^{*} \stackrel{*}{\Gamma}_{\lambda \beta}^{* \sigma}-b_{\alpha \beta} b_{\gamma}^{\sigma}\right. \\
& \left.+b_{\alpha \gamma} b_{\beta}^{\sigma}\right) \vec{a}_{\sigma}+\left({ }^{* \lambda} \Gamma_{\alpha \beta} b_{\gamma \lambda}--^{* \lambda}{ }_{\alpha \gamma} b_{\beta \lambda}+\partial_{\gamma} b_{\alpha \beta}-\partial_{\beta} b_{\alpha \gamma}\right)
\end{aligned}
$$

$$
\cdot \vec{a}_{3} \text {. }
$$

Since $\vec{a}_{\sigma}$ and $\vec{a}_{3}$ are linearly independent, we have

$$
\begin{array}{r}
\partial_{\gamma} \stackrel{*}{\Gamma}_{\alpha \beta}^{\sigma}-\partial_{\beta} \stackrel{*}{\Gamma}_{\alpha \gamma}^{\sigma}+\stackrel{*}{\Gamma}_{\alpha \beta}{ }_{\alpha \beta}^{* \sigma} \Gamma_{\lambda \gamma}-\stackrel{*}{\Gamma}_{\alpha \gamma}^{\lambda}{ }_{\alpha \gamma}^{* \sigma} \Gamma_{\lambda \beta}=b_{\alpha \beta} b_{\gamma}^{\sigma}-b_{\alpha \gamma} b_{\beta}^{\sigma}, \\
\partial_{\gamma} b_{\alpha \beta}-\stackrel{*}{\Gamma}_{\alpha \gamma}^{\lambda} b_{\beta \lambda}=\partial_{\beta} b_{\alpha \gamma}-\stackrel{*}{\Gamma}_{\alpha \beta}^{\lambda} b_{\gamma \lambda} .
\end{array}
$$

Thus, formula (41) has been proven.

\section{Examples}

3.1. Hemispherical Shell. Assume that the middle surface $S$ of shell is a hemispherical surface (see Figure 3 ) whose reference equation is given by the mapping $\vec{\theta}(\bar{\omega})$ defined by

$$
\vec{\theta}\left(y_{1}, y_{2}\right)=\left(r \cos y_{1} \sin y_{2}, r \sin y_{1} \sin y_{2}, r \cos y_{2}\right),
$$

where $r=1 \mathrm{~m}$ is the radius of the middle surface $S, 0 \leq y_{1} \leq$ $2 \pi$ is longitude, and $0 \leq y_{2} \leq \pi / 2$ is colatitude. The thickness of the middle surface $S$ is $2 \varepsilon$ where $\varepsilon$ is the semithickness.

Then,

$$
\begin{aligned}
\vec{a}_{1} & =\partial_{1} \vec{\theta}=\left(-r \sin y_{1} \sin y_{2}, r \cos y_{1} \sin y_{2}, 0\right), \\
\vec{a}_{2} & =\partial_{2} \vec{\theta} \\
& =\left(r \cos y_{1} \cos y_{2}, r \sin y_{1} \cos y_{2},-r \sin y_{2}\right), \\
\partial_{1} \vec{a}_{1} & =\left(-r \cos y_{1} \sin y_{2},-r \sin y_{1} \sin y_{2}, 0\right), \\
\partial_{1} \vec{a}_{2} & =\partial_{2} \vec{a}_{1}=\left(-r \sin y_{1} \cos y_{2}, r \cos y_{1} \cos y_{2}, 0\right), \\
\partial_{2} \vec{a}_{2} & =\left(-r \cos y_{1} \sin y_{2},-r \sin y_{1} \sin y_{2},-r \cos y_{2}\right) .
\end{aligned}
$$

Hence, the covariant and contravariant components of the metric tensor on $S$ are given by

$$
\begin{aligned}
& \left(a_{\alpha \beta}\right)=\left[\begin{array}{cc}
r^{2} \sin ^{2} y_{2} & 0 \\
0 & r^{2}
\end{array}\right], \\
& \left(a^{\alpha \beta}\right)=\left[\begin{array}{cc}
\frac{1}{r^{2} \sin ^{2} y_{2}} & 0 \\
0 & \frac{1}{r^{2}}
\end{array}\right] .
\end{aligned}
$$

Then,

$$
\begin{aligned}
\vec{a}_{3} & =\frac{\vec{a}_{1} \times \vec{a}_{2}}{\left|\vec{a}_{1} \times \vec{a}_{2}\right|} \\
& =\left(\cos y_{1} \sin y_{2}, \sin y_{1} \sin y_{2}, \cos y_{2}\right), \\
\partial_{1} \vec{a}_{3} & =\left(-\sin y_{1} \sin y_{2}, \cos y_{1} \sin y_{2}, 0\right), \\
\partial_{2} \vec{a}_{3} & =\left(\cos y_{1} \cos y_{2}, \sin y_{1} \cos y_{2},-\sin y_{2}\right), \\
\partial_{11} \vec{a}_{3} & =\left(-\cos y_{1} \sin y_{2},-\sin y_{1} \sin y_{2}, 0\right), \\
\partial_{12} \vec{a}_{3} & =\partial_{21} \vec{a}_{3}=\left(-\sin y_{1} \cos y_{2}, \cos y_{1} \cos y_{2}, 0\right), \\
\partial_{22} \vec{a}_{3} & =\left(-\cos y_{1} \cos y_{2},-\sin y_{1} \sin y_{2},-\cos y_{2}\right), \\
\left(b_{\alpha \beta}\right) & =\left[\begin{array}{cc}
-r \sin ^{2} y_{2} & 0 \\
0 & -r
\end{array}\right], \\
\left(c_{\alpha \beta}\right) & =\left[\begin{array}{cc}
\sin ^{2} y_{2} & 0 \\
0 & 1
\end{array}\right] .
\end{aligned}
$$

Thus,

$$
\begin{aligned}
& a=\operatorname{det}\left(a_{\alpha \beta}\right)=r^{4} \sin ^{2} y_{2}, \\
& b=\operatorname{det}\left(b_{\alpha \beta}\right)=r^{2} \sin ^{2} y_{2}, \\
& c=\operatorname{det}\left(c_{\alpha \beta}\right)=\sin ^{2} y_{2} .
\end{aligned}
$$

The Christoffel symbols on $S$ are as follows:

$$
\begin{aligned}
& \stackrel{*}{\Gamma}_{11,2}=-r^{2} \sin y_{2} \cos y_{2}, \\
& \stackrel{*}{\Gamma}_{12,1}=\stackrel{*}{\Gamma}_{21,1}=r^{2} \sin y_{2} \cos y_{2},
\end{aligned}
$$

other $\stackrel{*}{\Gamma}_{\alpha \beta, \gamma}=0$,

$$
\begin{aligned}
& *_{12}^{1}=*_{\Gamma}^{* 1}=\cot y_{2}, \\
& *_{11}^{2}=-\sin y_{2} \cos y_{2},
\end{aligned}
$$

other $\stackrel{*}{\Gamma}_{\beta \gamma}^{\alpha}=0$.

The Riemann tensors on $S$ are as follows:

$$
\begin{aligned}
& \stackrel{*}{R}_{1212}=\stackrel{*}{R}_{2121}=-r^{2} \sin ^{2} y_{2}, \\
& \stackrel{*}{R}_{1221}=\stackrel{*}{R}_{2112}=r^{2} \sin ^{2} y_{2},
\end{aligned}
$$

other $\stackrel{*}{R}_{\alpha \lambda \sigma \beta}=0$. 
Hence, for each $\varepsilon>0$, the reference configuration of the shell with middle surface $S=\vec{\theta}(\bar{\omega})$ is $\vec{\Theta}(\bar{\Omega})(\bar{\Omega}=\bar{\omega} \times[-\varepsilon, \varepsilon])$

$$
\vec{\Theta}(y, \xi)=\vec{\theta}(y)+\xi \vec{a}_{3}(y),
$$

where $-\varepsilon \leq \xi \leq \varepsilon$.

Therefore, the covariant and contravariant components of the metric tensor on $\vec{\Theta}(\bar{\Omega})$ are given by

$$
\begin{aligned}
& \left(g_{i j}\right)=\left[\begin{array}{ccc}
(r+\xi)^{2} \sin ^{2} y_{2} & 0 & 0 \\
0 & (r+\xi)^{2} & 0 \\
0 & 0 & 1
\end{array}\right], \\
& \left(g^{i j}\right)=\left[\begin{array}{ccc}
\frac{1}{(r+\xi)^{2} \sin ^{2} y_{2}} & 0 & 0 \\
0 & \frac{1}{(r+\xi)^{2}} & 0 \\
0 & 0 & 1
\end{array}\right] .
\end{aligned}
$$

The Christoffel symbols on $\vec{\Theta}(\bar{\Omega})$ are as follows:

$$
\begin{aligned}
\Gamma_{11,2} & =-(r+\xi)^{2} \sin y_{2} \cos y_{2}, \\
\Gamma_{11,3} & =-(r+\xi) \sin ^{2} y_{2}, \\
\Gamma_{12,1} & =\Gamma_{21,1}=(r+\xi)^{2} \sin y_{2} \cos y_{2}, \\
\Gamma_{13,1} & =(\xi-r) \sin ^{2} y_{2}, \\
\Gamma_{22,3} & =-(r+\xi), \\
\Gamma_{23,2} & =\xi-r, \\
\text { other } \Gamma_{i j, k} & =0, \\
\Gamma_{12}^{1} & =\Gamma_{21}^{1}=\cot y_{2}, \\
\Gamma_{13}^{1} & =\Gamma_{31}^{1}=\frac{1}{r+\xi}, \\
\Gamma_{11}^{2} & =-\sin y_{2} \cos y_{2}, \\
\Gamma_{23}^{2} & =\Gamma_{32}^{2}=\frac{1}{r+\xi}, \\
\Gamma_{11}^{3} & =-(r+\xi) \sin ^{2} y_{2}, \\
\Gamma_{22}^{3} & =-(r+\xi), \\
\text { other } \Gamma_{i j}^{k} & =0 .
\end{aligned}
$$

The Riemann tensors on $\vec{\Theta}(\bar{\Omega})$ are as follows:

$$
\begin{aligned}
R_{i k j}^{p} & =0, \\
R_{i l k j} & =0,
\end{aligned}
$$$$
i, j, k, p, l=1,2,3 \text {. }
$$

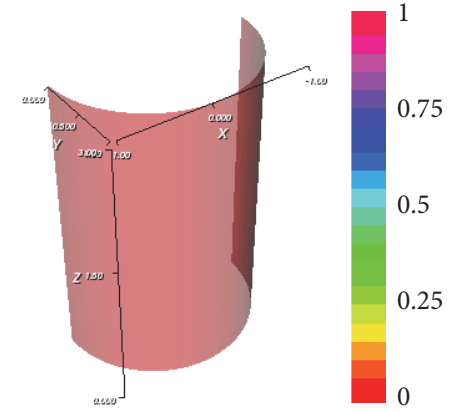

Figure 4: Middle surface of semicylindrical shell.

3.2. Semicylindrical Shell. Assume that the middle surface $S$ of shell is a semicylindrical surface (see Figure 4) whose reference equation is given by the mapping $\vec{\theta}(\bar{\omega})$ defined by

$$
\vec{\theta}\left(y_{1}, y_{2}\right)=\left(r \cos y_{1}, r \sin y_{1}, y_{2}\right),
$$

where $r=1 \mathrm{~m}$ is a constant, $0 \leq y_{1} \leq \pi$, and $0 \leq y_{2} \leq h(h=$ $3 \mathrm{~m}$ ). The thickness of the middle surface $S$ is $2 \varepsilon$ where $\varepsilon$ is the semithickness.

Then,

$$
\begin{aligned}
\vec{a}_{1} & =\partial_{1} \vec{\theta}=\left(-r \sin y_{1}, r \cos y_{1}, 0\right), \\
\vec{a}_{2} & =\partial_{2} \vec{\theta}=(0,0,1), \\
\partial_{1} \vec{a}_{1} & =\left(-r \cos y_{1},-r \sin y_{1}, 0\right), \\
\partial_{1} \vec{a}_{2} & =\partial_{2} \vec{a}_{1}=(0,0,0), \\
\partial_{2} \vec{a}_{2} & =(0,0,0) .
\end{aligned}
$$

Therefore, the covariant and contravariant components of the metric tensor on $S$ are given by

$$
\begin{aligned}
& \left(a_{\alpha \beta}\right)=\left[\begin{array}{ll}
r^{2} & 0 \\
0 & 1
\end{array}\right], \\
& \left(a^{\alpha \beta}\right)=\left[\begin{array}{cc}
r^{-2} & 0 \\
0 & 1
\end{array}\right] .
\end{aligned}
$$

Then,

$$
\begin{aligned}
\vec{a}_{3} & =\frac{\vec{a}_{1} \times \vec{a}_{2}}{\left|\vec{a}_{1} \times \vec{a}_{2}\right|}=\left(\cos y_{1}, \sin y_{1}, 0\right), \\
\partial_{1} \vec{a}_{3} & =\left(-\sin y_{1}, \cos y_{1}, 0\right), \\
\partial_{2} \vec{a}_{3} & =(0,0,0), \\
\partial_{11} \vec{a}_{3} & =\left(-\cos y_{1},-\sin y_{1}, 0\right),
\end{aligned}
$$




$$
\begin{aligned}
& \partial_{12} \vec{a}_{3}=\partial_{21} \vec{a}_{3}=(0,0,0), \\
& \partial_{22} \vec{a}_{3}=(0,0,0), \\
& \left(b_{\alpha \beta}\right)=\left[\begin{array}{ll}
-r & 0 \\
0 & 0
\end{array}\right], \\
& \left(c_{\alpha \beta}\right)=\left[\begin{array}{ll}
1 & 0 \\
0 & 0
\end{array}\right] .
\end{aligned}
$$

Thus,

$$
\begin{aligned}
& a=\operatorname{det}\left(a_{\alpha \beta}\right)=r^{2}, \\
& b=\operatorname{det}\left(b_{\alpha \beta}\right)=r^{2}, \\
& c=\operatorname{det}\left(c_{\alpha \beta}\right)=1 .
\end{aligned}
$$

The Christoffel symbols on $S$ are

$$
\begin{gathered}
\stackrel{*}{\Gamma}_{\alpha \beta, \gamma}=0, \\
\stackrel{*}{\Gamma} \alpha_{\beta \gamma}=0 .
\end{gathered}
$$

The Riemann tensors on $S$ are as follows:

$$
\begin{aligned}
& \stackrel{*}{R}_{\alpha \gamma \beta}^{\sigma}=0, \\
& \stackrel{*}{R}_{\alpha \lambda \gamma \beta}=0, \\
& \quad \alpha, \beta, \sigma, \gamma, \lambda=1,2 .
\end{aligned}
$$

Hence, for each $\varepsilon>0$, the reference configuration of the shell with middle surface $S=\vec{\theta}(\bar{\omega})$ is $\vec{\Theta}(\bar{\Omega})(\bar{\Omega}=\bar{\omega} \times[-\varepsilon, \varepsilon])$

$$
\vec{\Theta}(y, \xi)=\vec{\theta}(y)+\xi \vec{a}_{3}(y),
$$

where $-\varepsilon \leq \xi \leq \varepsilon$.

So, the covariant and contravariant components of the metric tensor on $\vec{\Theta}(\bar{\Omega})$ are given by

$$
\begin{aligned}
& \left(g_{i j}\right)=\left[\begin{array}{ccc}
(r+\xi)^{2} & 0 & 0 \\
0 & 1 & 0 \\
0 & 0 & 1
\end{array}\right], \\
& \left(g^{i j}\right)=\left[\begin{array}{ccc}
\frac{1}{(r+\xi)^{2}} & 0 & 0 \\
0 & 1 & 0 \\
0 & 0 & 1
\end{array}\right] .
\end{aligned}
$$

The Christoffel symbols on $\vec{\Theta}(\bar{\Omega})$ are as follows:

$$
\begin{aligned}
\Gamma_{11,3} & =-(r+\xi), \\
\Gamma_{13,1} & =(r+\xi), \\
\Gamma_{i j, k} & =0, \\
\Gamma_{13}^{1} & =\Gamma_{31}^{1}=\frac{1}{r+\xi}, \\
\Gamma_{11}^{3} & =-(r+\xi),
\end{aligned}
$$$$
\text { other } \Gamma_{i j, k}=0 \text {, }
$$

$$
\text { other } \Gamma_{i j}^{k}=0 \text {. }
$$

The Riemann tensors on $\vec{\Theta}(\bar{\Omega})$ are as follows:

$$
\begin{aligned}
R_{i k j}^{p} & =0, \\
R_{i l k j} & =0, \\
i, j, k, p, l & =1,2,3 .
\end{aligned}
$$

\section{Competing Interests}

There are no competing interests regarding this paper.

\section{Acknowledgments}

This paper is supported by National Natural Science Foundation of China (NSFC 11571275, NSFC 11572244) and Program of Industry in Shaanxi Province (2015GY021).

\section{References}

[1] P. G. Ciarlet, An Introduction to Differential Geometry with Applications to Elasticity, Springer, Heidelberg, Germany, 2005.

[2] P. G. Ciarlet, Mathematical Elasticity, vol. 3 of Theory of Shells, North-Holland, 2000.

[3] B. E. Pobedrya, Lectures on Tensor Analysis, MGU, Moscow, Russia, 1986 (Russian).

[4] I. N. Vekua, The Basics of Tensor Analysis and Theory of Covariants, Nauka, Moscow, Russia, 1978 (Russian).

[5] M. U. Nikabadze, "On some problems of tensor calculus. I," Journal of Mathematical Sciences, vol. 161, no. 5, pp. 668-697, 2009.

[6] W. T. Koiter, "A consistent first approximation in the general theory of thin elastic shells," in Proceedings of the IUTAM Symposium on the Theory of Thin Elastic Shells, pp. 12-33, Delft, The Netherlands, August 1959.

[7] W. T. Koiter, "On the foundations of the linear theory of thin elastic shells," Proceedings of the Koninklijke Nederlandse Akademie van Wetenschappen B, vol. 73, pp. 169-195, 1970.

[8] K. Li and X. Shen, "A dimensional splitting method for the linearly elastic shell," International Journal of Computer Mathematics, vol. 84, no. 6, pp. 807-824, 2007.

[9] X. Shen, K. Li, and Y. Ming, "Asymptotic expansions of stress tensor for linearly elastic shell," Applied Mathematical Modelling, vol. 37, no. 16-17, pp. 7964-7972, 2013.

[10] K. Li and A. Huang, Tensor Analysis and Its Applications, Chinese Scientific Press, 2004 (Chinese). 
[11] L. Xiao, "Justification of two-dimensional nonlinear dynamic shell equations of Koiter's type," Nonlinear Analysis: Theory, Methods \& Applications, vol. 62, no. 3, pp. 383-395, 2005.

[12] P. G. Ciarlet, Differential Geometry: Theory and Applications, Higher Education Press, Shanghai, China, 2007. 


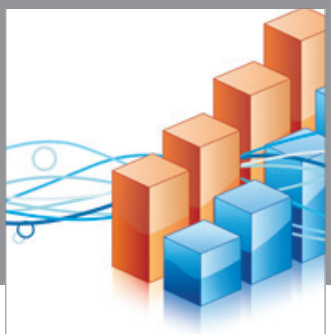

Advances in

Operations Research

vatem alat4

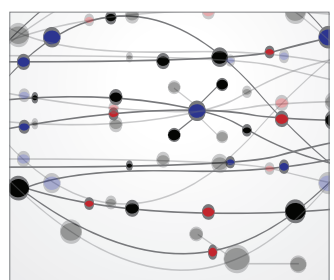

\section{The Scientific} World Journal
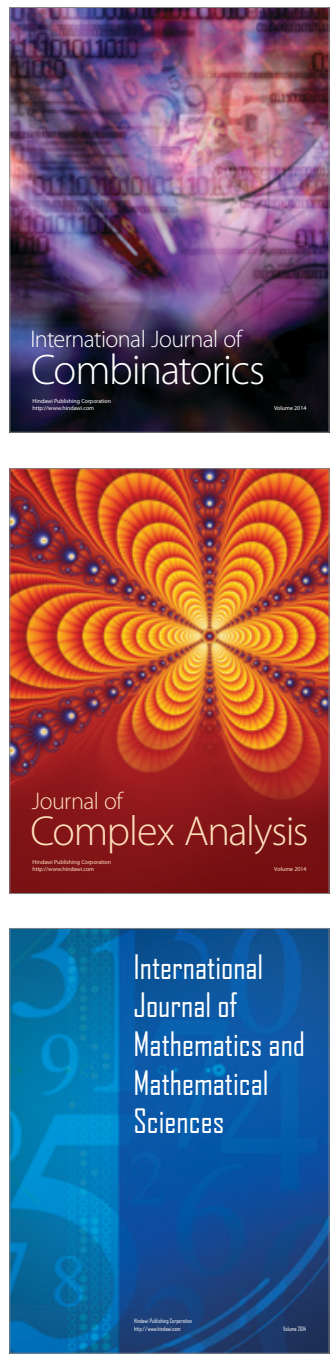
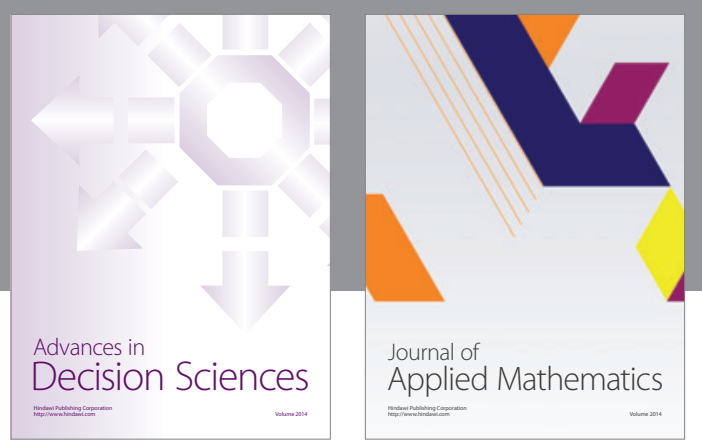

Algebra

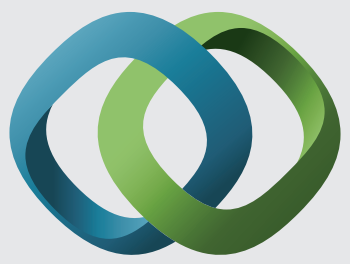

\section{Hindawi}

Submit your manuscripts at

http://www.hindawi.com
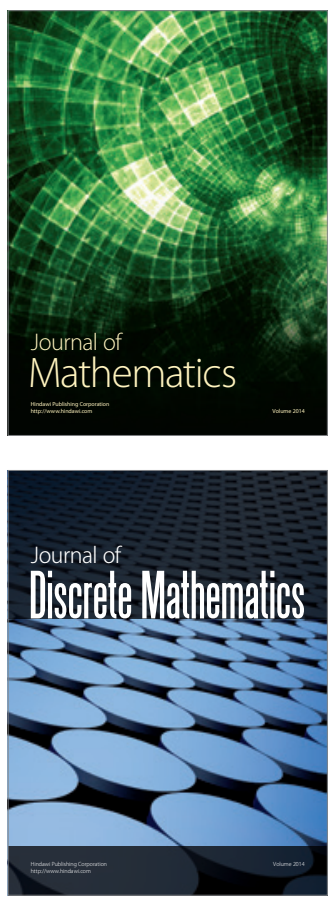

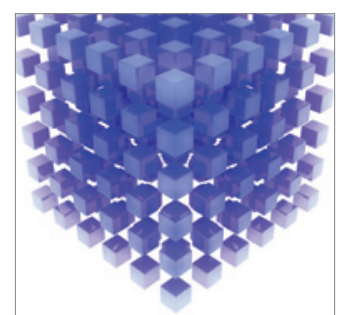

Mathematical Problems in Engineering
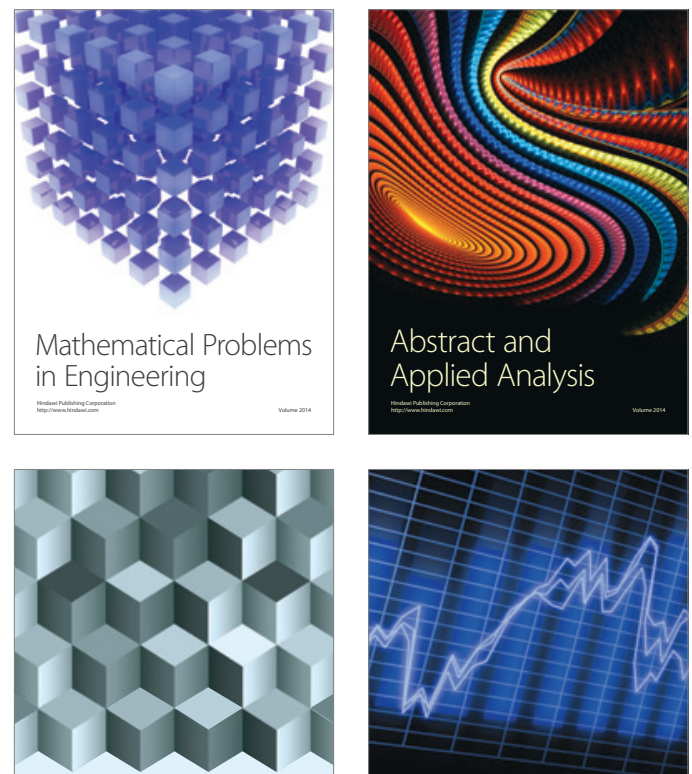

Journal of

Function Spaces

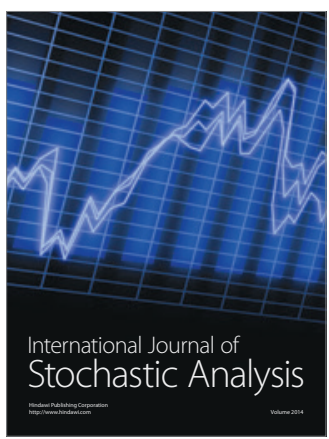

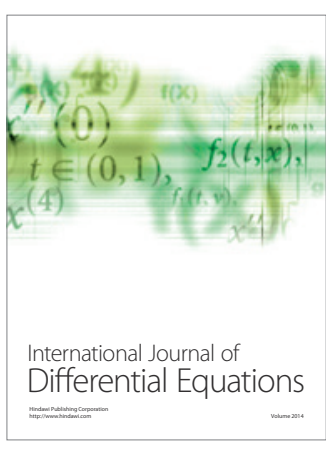
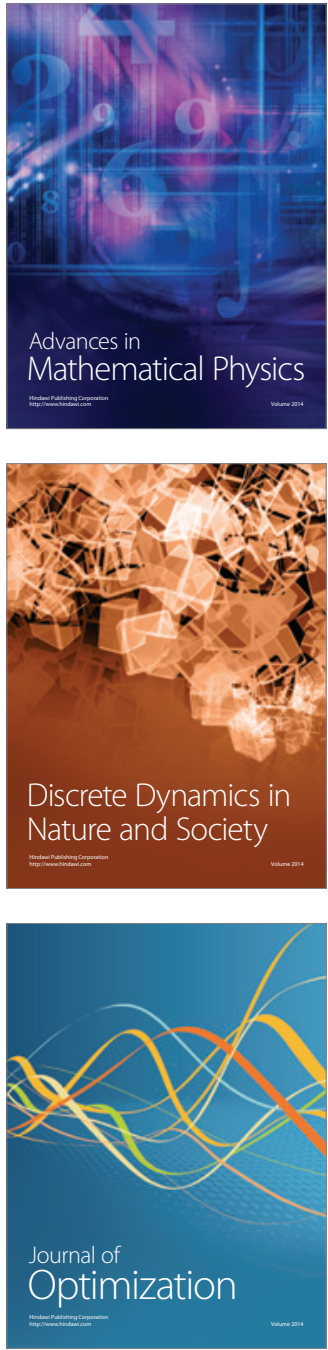